\title{
Stanford Cancer Institute
}

National Cancer Institute

\section{Source}

National Cancer Institute. Stanford Cancer Institute. NCI Thesaurus. Code C105626.

The Stanford Cancer Institute is focused on improving the diagnosis, treatment, and outcomes for cancer patients, understanding cancer etiologies among diverse populations, and reducing the incidence of cancer. It became an $\mathrm{NCl}$-designated cancer center in 2007. It is a Stanford School of Medicine center. 